\title{
Mutants of Aerobacter aerogenes Blocked in the Accumulation of Inorganic Polyphosphate
}

\author{
By RUTH L. HAROLD AND F. M. HAROLD \\ Department of Experimental Chemistry, Division of Research and Laboratories, \\ the National Jervish Hospital at Denver, Colorado, U.S.A.
}

(Received 10 September 1962)

SUMMARY

Mutants of Aerobacter aerogenes blocked in the accumulation of inorganic polyphosphate were isolated by the following method. Organisms previously subjected to ultraviolet (u.v.) irradiation were induced to accumulate polyphosphate in medium containing ${ }^{32} \mathrm{P}$. Upon subsequent growth in medium devoid of phosphate, RNA and DNA synthesis took place at the expense of polyphosphate. The organisms containing radioactive DNA were inactivated by decay of the ${ }^{32} \mathrm{P}$ during prolonged cold storage. From the survivors, mutants unable to form volutin granules were selected by microscopic examination. The mutants fell into two classes. The majority did not accumulate polyphosphate when phosphate was added to a phosphate-starved culture, but did so upon prolonged sulphur starvation. The remainder of the mutants did not accumulate polyphosphate under either condition. Both kinds of mutant grew well in a defined medium and showed no obvious physiological disabilities.

\section{INTRODUCTION}

Bacteria from cultures whose growth has ceased as a result of certain kinds of nutrient imbalance often contain prominent intracellular granules characterized by metachromatic staining with basic dyes and by their opacity in the electron microscope. These 'volutin granules' have been identified as deposits of inorganic polyphosphate (reviewed by Wilkinson \& Duguid, 1960; Kuhl, 1960). The nature of volutin granules is controversial. While most investigators regard them as distinct entities, some continue to identify them with nuclei or with mitochondrial equivalents. Their composition has been claimed to include ribonucleic acid (RNA), protein, lipids and various metal ions (Widra, 1959) in addition to polyphosphate. The function of polyphosphate granules likewise remains to be clarified. They have been considered to be storage deposits of phosphorus (Wiame, 1949), highenergy phosphate (Hoffmann-Ostenhof \& Weigert, 1952) or divalent cations (Katchman \& Fetty, 1955). Inorganic polyphosphate has also been assigned a specific role in cell division (Sall, Mudd \& Takagi, 1958; Scherbaum, 1960) and in the control of energy metabolism through ATP dissipation (Harold, 1962).

Mutants genetically blocked in various phases of polyphosphate metabolism should provide incisive tools for the resolution of these controversies. It is the purpose of the present paper to describe a method for the isolation of such mutants and to report preliminary investigations of their physiology. The principle used was the inactivation of bacteria by the decay of ${ }^{32} \mathrm{P}$ incorporated into their deoxy- 
ribonucleic acid (DNA) (Fuerst \& Stent, 1956). Ultraviolet-irradiated Aerobacter aerogenes organisms were induced to accumulate polyphosphate in medium containing radioactive phosphate. Upon subsequent transfer to medium devoid of phosphate, RNA and DNA synthesis took place at the expense of the accumulated radioactive polyphosphate. The organisms were then collected and stored in solid $\mathrm{CO}_{2}$ for several weeks. Decay of the ${ }^{32} \mathrm{P}$ incorporated into DNA inactivated most of the organisms. Any mutants unable to accumulate polyphosphate would not have incorporated ${ }^{32} \mathrm{P}$ into their DNA and would thus be expected to survive. The survivors did in fact include up to $5 \%$ of mutants blocked in the accumulation of polyphosphate.

\section{METHODS}

Organism. Aerobacter aerogenes strain A3(0) was supplied by Dr J. F. Wilkinson. A clone of organisms showing good polyphosphate accumulation was picked and used in all subsequent experiments.

Growth experiments. Two types of growth medium were employed: (a) W medium designates the medium of Smith, Wilkinson \& Duguid (1954), containing $2300 \mu \mathrm{g}$. $\mathrm{Pi} / \mathrm{ml}$. as phosphorus source and buffer. (b) $\mathrm{T}_{\text {subscript }}$ media are a modification of this medium containing tris buffer (10 g./l., $\mathrm{pH} 7 \cdot 6)$; the subscript indicates the phosphorus content in $\mu \mathrm{g}$. phosphorus $/ \mathrm{ml}$.

All cultures were grown at $37^{\circ}$ on a rotary shaker. Viable counts were performed by plating on $\mathrm{W}$ medium. The optical density of suspensions of organisms was estimated turbidimetrically at $600 \mathrm{~m} \mu$. An optical density value at $600 \mathrm{~m} \mu$ (OD 600) of 1.0 corresponds to $7 \times 10^{8}$ organisms $/ \mathrm{ml}$.

Polyphosphate accumulation was induced by two distinct procedures described by Smith et al. (1954): (i) Bacteria were grown overnight on W medium, centrifuged, washed with phosphate or tris buffer, resuspended in medium devoid of sulphate, and incubated for $6 \mathrm{hr}$. (ii) An overnight culture was prepared in $\mathrm{T}_{1 \cdot 8}$ medium, growth being limited by the available phosphate. Next morning glucose and phosphate were added and the culture incubated for $1 \mathrm{hr}$. Alternatively, organisms from an overnight culture in $\mathrm{W}$ medium were centrifuged, washed and incubated overnight in $\mathrm{T}_{\mathbf{0}} \cdot \mathbf{4}$ medium. Addition of glucose and phosphate then induced excellent accumulation of polyphosphate within $1 \mathrm{hr}$.

Analytical methods. Samples (20 ml.) were withdrawn and the organisms centrifuged down. Fractionation of the organisms and estimation of inorganic polyphosphate was carried out by methods described earlier (Harold, 1960). Briefly, the organisms were extracted in succession with cold perchloric acid (PCA; $0.5 \mathrm{~N}$; 15 min.), ethanol, ethanol + ether and hot PCA $\left(0.5 \mathrm{~N} ; 15 \mathrm{~min}\right.$. at 70 $\left.{ }^{\circ}\right)$. Most of the polyphosphate was found in the hot PCA extract ('insoluble polyphosphate'); it was estimated as acid-labile phosphate after removal of nucleic acid by adsorption on Norit A. Organisms subjected to procedure (ii) also contained soluble polyphosphate in the cold PCA extract. This was determined as acid-labile phosphate after removal of nucleotides with Norit $A$.

Chemicals. Carrier-free ${ }^{32} \mathrm{P}$ was purchased from Volk Radiochemical Co., Skokie, Ill., U.S.A.

Isolation of mutants. An overnight culture of Aerobacter aerogenes A3(0) grown in $\mathrm{W}$ medium was harvested and the organisms resuspended in tris-buffer to OD 600 of $0 \cdot 10$. Ten ml. of this suspension were u.v.-irradiated with a Mineralight V-41 
lamp for a period of time sufficient to decrease the viable count by $99.9 \%$. The irradiated organisms were then diluted tenfold in $\mathrm{W}$ medium or in nutrient broth and incubated for 10-18 hr. These and all subsequent operations were carried out under aseptic conditions. The organisms were then harvested by centrifugation, washed once with tris buffer and resuspended in $T_{0 \cdot 4}$ medium at an OD 600 value of $0 \cdot 1-0 \cdot 3$. After incubation for $18 \mathrm{hr}$. the organisms were collected and resuspended in $\mathrm{T}_{0}$ medium at $\mathrm{OD} 600$ about $0 \cdot 8$. A sample $(0 \cdot 20 \mathrm{ml}$.) of this suspension was pipetted into a dry tube containing $3 \mu \mathrm{g}$. phosphorus and $300 \mu \mathrm{c} .{ }^{32} \mathrm{P}$ (as $\mathrm{KH}_{2} \mathrm{PO}_{4}$ ). The tube was then incubated for $1 \mathrm{hr}$. at $37^{\circ}$ to permit accumulation of radioactive polyphosphate by the wild-type organisms. At the end of this period the organisms were centrifuged down, washed twice with $T_{0}$ medium and resuspended in $\mathbf{0 . 4 0} \mathrm{ml}$. of $\mathrm{T}_{0}$ medium. They were then incubated for $4 \mathrm{hr}$. at $37^{\circ}$ to permit growth and nucleic acid synthesis by the wild-type organisms at the expense of accumulated ${ }^{32} P$. polyphosphate. Finally the culture was diluted tenfold with $\mathrm{T}_{\mathbf{0}}$ medium, and glycerol added to a final concentration of $10 \%$. Samples $(1 \mathrm{ml}$.) were dispensed into screw-cap test tubes. These were frozen in solid $\mathrm{CO}_{2}+$ methylcellosolve and stored in solid $\mathrm{CO}_{2}$.

At intervals these tubes were thawed at $37^{\circ}$ and appropriate dilutions plated on W medium. As shown in Fig. 1 the viable count decreased exponentially, levelling out after about 1 week with about $0.01 \%$ of the organisms surviving. Most of the survivors were identical with the parent strain with respect to polyphosphate accumulation; the mutants, which constituted $2-5 \%$ of the population, were picked out by microscopic screening as follows. Colonies to be screened were replated on $W$ medium. Simultaneously, part of each colony was transferred to $2.5 \mathrm{ml}$. of $\mathrm{T}_{0 \cdot 4}$ medium and incubated with shaking overnight. Next morning glucose and phosphate $\left(100 \mu \mathrm{g} . / \mathrm{ml}\right.$.) were added to each tube. After $1 \mathrm{hr}$. at $37^{\circ}$ the organisms were sedimented, a sample smeared on a microscope slide, heat fixed and stained for volution granules by Laybourn's procedure (1924). Clones which did not show volutin granules were thus detected; in general they turned out to be polyphosphateless mutants on subsequent investigation. Such potential polyphosphateless mutants were repurified and tested for polyphosphate accumulation. The extent of polyphosphate accumulation was determined chemically or by the use of ${ }^{32} \mathrm{P}$.

\section{RESULTS}

By the techniques described in the previous section, some 25 clones were isolated from three separate u.v.-irradiations. All were incapable of accumulating 'polyphosphate when radioactive phosphate was added to a culture previously subjected to phosphorus starvation. Two clones from each u.v. irradiation were compared with the parent strain with respect to typical reactions of Aerobacter aerogenes (Wilkinson, Duguid \& Edmonds, 1954). The Gram reaction and motility, fermentation of sucrose, lactose, mannitol, glycerol and inositol, colony appearance on eosine methylene blue agar, citrate utilization and production of indole and acetylmethylcarbinol, were all like the reactions given by Aerobacter aerogenes A3(0). The mutants, like $A$. aerogenes A3(0), produced neither capsule nor loose slime.

The patterns of phosphorus metabolism in mutant and wild-type organisms were compared in growth experiments. Cultures whose growth was limited by phosphorus deficiency were prepared by overnight growth in $\mathbf{T}_{1 \cdot 8}$ medium. These organisms were then harvested and resuspended in $\mathrm{T}_{230}$ medium. Under these 


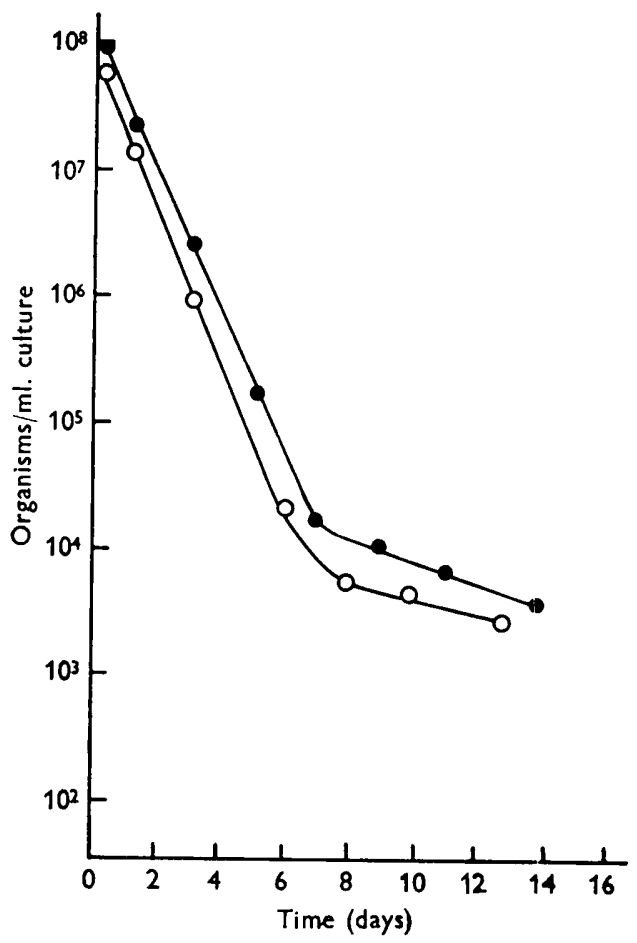

Fig. 1

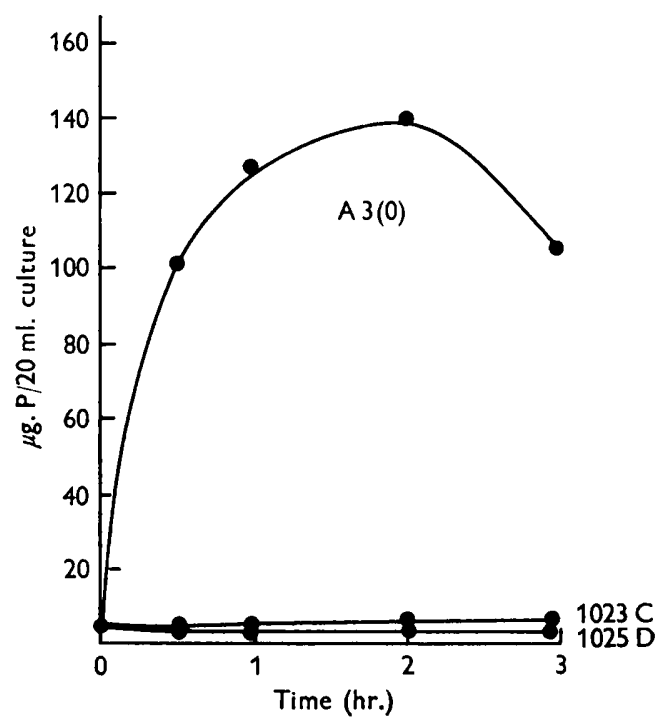

Fig. 2

Fig. 1. Inactivation of Aerobacter aerogenes containing ${ }^{32} \mathrm{P}$ - labelled nucleic acids upon storage in solid $\mathrm{CO}_{2}$. For details of experiments see text. The open and solid circles represent separate experiments.

Fig. 2. Accumulation of inorganic polyphosphate in wild-type Aerobacter aerogenes A 3(0) and mutants $1023 \mathrm{~B}$ and $1025 \mathrm{D}$. Overnight cultures were prepared in $\mathrm{T}_{1.8}$ medium. Phosphate and glucose were added at $0 \mathrm{hr}$.
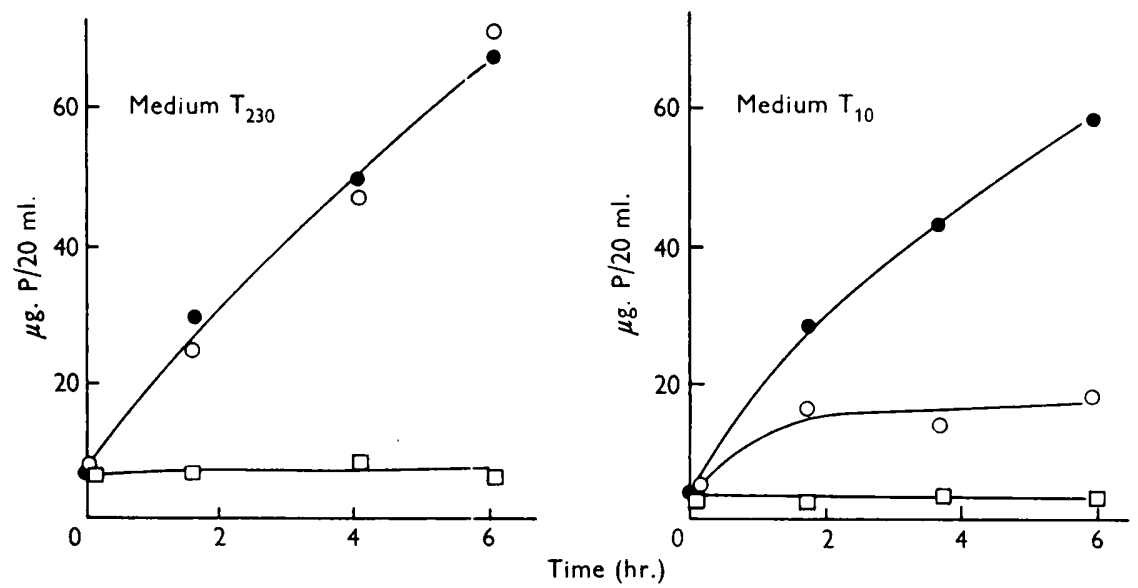

Fig. 3. Accumulation of inorganis: polyphosphate in wild-type Aerobacter aerogenes $A 3(0)$ and mutants $1023 B$ and $1025 D$ when sulphur starved. Organisms from overnight cultures in ' $\mathrm{T}_{230}$ medium were resuspended in sulphur-deficient media containing 230 or $10 \mu \mathrm{g} . / \mathrm{ml}$. P at $0 \mathrm{hr}$. $\longrightarrow=$ Strain A3(0); $\bigcirc-\mathrm{O}=$ mutant $1025 \mathrm{D} ; \square-\square$, = mutant $1023 \mathrm{~B}$. 
conditions there was rapid accumulation of polyphosphate in strain $\mathbf{A 3 ( 0 )}$ but none in any of the mutants (Fig. 2). This sharp difference in pattern was independent of the amount of phosphorus added to the starved cultures and of the duration of starvation.

In other experiments, the response of the wild-type and mutant organisms was compared in a sulphur deficient medium. Cultures were grown overnight on $\mathrm{T}_{230}$ medium; the organisms were collected, washed and resuspended in $\mathrm{T}$ media devoid of sulphur. Under these conditions wild-type organisms exhibited a marked accumulation of polyphosphate, regardless of the concentration of phosphorus in the medium. The mutants fell into two classes: two clones (which might have been progeny of a single mutational event), 1023B and $1023 \mathrm{C}$, accumulated no polyphosphate upon sulphur starvation in any medium tried. There was, however, a small variable increase in the concentration of orthophosphate and of acid-soluble organic phosphate. The remainder of the mutants were capable of accumulating polyphosphate upon sulphur starvation, but only in media of relatively high phosphorus content. Accumulation was poor on medium containing only $10 \mu \mathrm{g}$. radioactive phosphate (Fig. 3).

Thus far, only preliminary attempts have been made to determine whether the polyphosphateless mutants suffer any physiological disabilities as a result of their inability to accumulate polyphosphate. All of the mutants tested were identical with the parent strain with respect to growth rate and growth yield in $W$ and $T_{230}$ media. Also there was no difference in the length of the lag period which preceded resumption of growth of sulphur- or phosphorus-starved cultures when the missing nutrient was supplied.

\section{DISCUSSION}

The principle underlying our method for the selection of mutants with defective polyphosphate metabolism is the decay of ${ }^{32} \mathrm{P}$ incorporated into DNA; there is no rigorous proof that the inactivation of the wild-type population was indeed due to this process. However, the proposed mechanism is supported by the exponential inactivation curve (Fig. 1) and by preliminary experiments which showed the transfer of phosphorus from polyphosphate to both DNA and RNA under the conditions used. While our method has undoubtedly permitted the isolation of mutants blocked in the accumulation of polyphosphate, a measure of selection may well be inherent in it. If a genetic block in polyphosphate metabolism is such as to be lethal to the organism, or to prevent its multiplication in the medium used, the mutant would be lost. Consequently the only mutants recovered will be those which exhibit non-lethal modifications of polyphosphate metabolism. It would thus be not too surprising if all the mutants so isolated grew well in minimal medium, despite their inability to accumulate polyphosphate. In the absence of information about the enzymic capacities of our mutants, failure to accumulate polyphosphate need not indicate a block in polyphosphate synthesis, since a high turnover rate might prevent net accumulation. The information available so far is therefore not incompatible with a vital role for polyphosphate in cellular economy, provided that its function can be discharged by trace amounts of it. For instance, a catalytic concentration of polyphosphate would suffice for the dissipation of ATP via the polyphosphate cycle (Harold, 1962). However, the very existence of mutants such as $1023 \mathrm{~B}$, which appears to be incapable of accumulating 
polyphosphate under any conditions, argues that volutin granules and the capacity to form a large polyphosphate pool are dispensable functions. (The fact that volutin granules were not observed in our mutants supports their identification as deposits of inorganic polyphosphate.) We have here evidence against the obligatory participation of a large polyphosphate pool in a vital process, such as cell division (Scherbaum, 1960). The nature of the enzymic defects which prevent polyphosphate accumulation in the mutants is under investigation. However, we may anticipate two distinct blocks: one which prevents polyphosphate accumulation altogether, while the other permits the slow accumulation seen in sulphur starvation but which prevents the dramatic burst normally observed when radioactive phosphate is restored to a phosphorus-starved culture. The induction of polyphosphate accumulation by these two procedures thus appears to reflect distinct and separable metabolic processes.

It is a pleasure to thank Professor G. S. Stent for suggestions about the selection technique described. We are grateful to Miss Norma Layher for skilful assistance. This investigation was supported in part by a research grant (E-3568) from the National Institutes of Health, Public Health Service, U.S.A.

\section{REFERENCES}

Fuerst, C. R. \& Stent, G. S. (1956). Inactivation of bacteria by decay of incorporated radioactive phosphorus. J. gen. Physiol. 40, 73.

HAROLD, F. M. (1960). Accumulation of inorganic polyphosphate in mutants of Neurospora crassa. Biochim. biophys. Acta, 45, 172.

Harold, F. M. (1962). Depletion and replenishment of the inorganic polyphosphate pool in Neurospora crassa. J. Bact. 83, 1047.

Hoffmann-Ostenhof, O. \& Weigert, W. (1952). Über die mögliche Funktion des polymeren Metaphosphats als Speicher energiereichen Phosphats in der Hefe. Naturwissenschaften, 38, 303.

Katchman, B. J. \& Fetty, W. O. (1955). Phosphorus metabolism in growing cultures of Saccharomyces cerevisiae. J. Bact. 69, 607.

Kunl, A. (1960). Die Biologie der kondensierten anorganischen Phosphate. Ergebn. Biol. 23, 144.

Laybourn, R. L. (1924). A modification of Albert's stain for the diphtheria bacillus. J. Amer. med. Ass. 83, 121.

Sall, T., Mudd, S. \& TAKagi, A. (1958). Phosphate accumulation and utilization as related to synchronized cell division of Corynebacterium diphtheriae. J. Bact. 76, 640 .

Scherbaum, O. H. (1960). Synchronous division of microorganisms. Annu. Rev. Microbiol. 14, 283.

Smith, I. W., Wilkinson, J. F. \& Duguid, J. P. (1954). Volutin production in Aerobacter aerogenes due to nutrient imbalance. $J$. Bact. 68, 450 .

Wiame, J. M. (1949). The occurrence and physiological behavior of two metaphosphate fractions in yeast. $J$. biol. Chem. 178, 919 .

WidrA, A. (1959). Metachromatic granules of microorganisms. J. Bact. 78, 664.

Wrlkinson, J. F., Duguin, J. P. \& Edmonds, P. N. (1954). The distribution of polysaccharide production in Aerobacter and Escherichia strains and its relation to antigenic character. J. gen. Microbiol. 11, 59.

Wilkinson, J. F. \& Duguid, J. P. (1960). The influence of cultural conditions on bacterial cytology. Int. Rev. Cytol. 9, 1.

Note added in proof: Isolation of mutants which failed to accumulate polyphosphate following phosphorus starvation has now been repeated, using a methioninerequiring mutant of Aerobacter aerogenes A3 (0). 